\title{
Insulin autoimmune syndrome - time to remember
}

\author{
Nalan Okuroglu', Meltem Sertbas' ${ }^{1}$, Cafer Akkoz', Yasar Sertbas ${ }^{1}$, Seda Sancak², Ali Ozdemir ${ }^{1}$ \\ ${ }^{1}$ Department of Internal Medicine, Fatih Sultan Mehmet Education and Research Hospital, Istanbul, Turkey \\ ${ }^{2}$ Department of Endocrinology, Fatih Sultan Mehmet Education and Research Hospital, Istanbul, Turkey
}

Key words: HLA-DR typing; hypoglycaemia; insulin autoantibodies; insulin autoimmune syndrome

Insulin autoimmune syndrome (IAS) is characterised by spontaneous hypoglycaemia, and excessive serum insulin levels and insulin autoantibodies, which occur in the absence of exogenous insulin. In particular, it is seen with drugs containing the sulfhydryl group. Alpha lipoic acid (ALA) is a sulfhydryl-containing antioxidant used for diabetic neuropathy, non-neuropathic pain, and also as anti-ageing and as a dietary supplement. Although IAS is frequently reported in the Asian population due to its genetic predisposition, it is rarely seen in the Caucasian race, as in Turkey. Herein, we report a case diagnosed as IAS after using ALA with vitamin B due to pain.

A 62-year-old woman referred to our clinic for experiencing hypoglycaemia episodes. Her complaints were over the preceding two weeks including recurrent dizziness, malaise, and fatigue. She had hypertension and chronic obstructive lung disease for which she was using telmisartan/HCT and budesonide plus formoterol. No previous diagnosis of diabetes or insulin administration was detected.

At admission, informed consent form was obtained. Her body weight was $86 \mathrm{~kg}$, height $155 \mathrm{~cm}$, and body mass index (BMI) $35.8 \mathrm{~kg} / \mathrm{m}^{2}$. Fasting plasma glucose was $59 \mathrm{mg} / \mathrm{dL}$, C-peptide was measured as $6.1 \mathrm{ng} / \mathrm{mL}$, insulin $>600 \mathrm{uU} / \mathrm{mL}$, and $\mathrm{HbA}_{1 \mathrm{c}} 6.3 \%$. Screening tests for primary and secondary adrenal insufficiency were performed; early morning cortisol and adrenocorticotropin (ACTH) values were 18.4 and $13.8 \mathrm{ug} / \mathrm{dl}$, respectively. Laboratory tests at admission are listed in Table 1. She was hospitalised for 72 hours. In fasting test no hypoglycaemia was detected. The 72-hour fasting test result is shown in Figure 1.

After detecting endogenous hyperinsulinaemia we performed magnetic resonance imaging (MRI) to exclude insulinoma. MRI revealed an increased size of liver by $195 \mathrm{~mm}$ but no mass lesion of the pancreas. Consequently, regarding IAS, insulin autoantibodies were measured.
Table I. Result of the laboratory test at admission

\begin{tabular}{lcc}
\hline Parameters & & Reference range \\
\hline WBC [103/uL] & 5.11 & $4-10$ \\
\hline Hgb [g/dl] & 11.2 & $11-15$ \\
\hline HCT (\%) & 34.2 & $36-47$ \\
\hline PLT [103/uL] & 233 & $100-400$ \\
\hline Glucose [mg/dl] & 59 & $\begin{array}{c}<70 ~ m g \\
\text { hypoglycaemia }\end{array}$ \\
\hline C-peptide [ng/mL] & 6.1 & $0.90-7.1$ \\
\hline Insulin [uU/ml] & $>600$ & $1.9-23$ \\
\hline
\end{tabular}

WBC — white blood cells; Hgb — haemoglobin; HCT — hematocrit; PLT — platelets

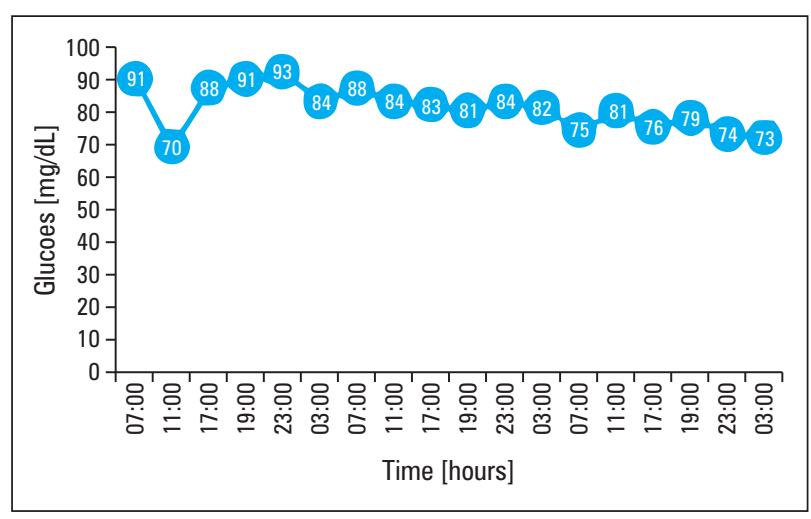

Figure 1. 72-hour fasting plasma glucose levels

The tests showed very high insulin levels and insulin antibodies, and so IAS was diagnosed. She was prescribed $600 \mathrm{mg} /$ daily ALA due to shoulder pain a month earlier, and her symptoms began at the end of the ALA treatment.

She was advised to avoid ALA, eat small meals but frequently, eat low-carbohydrate foods, and avoid fasting. 


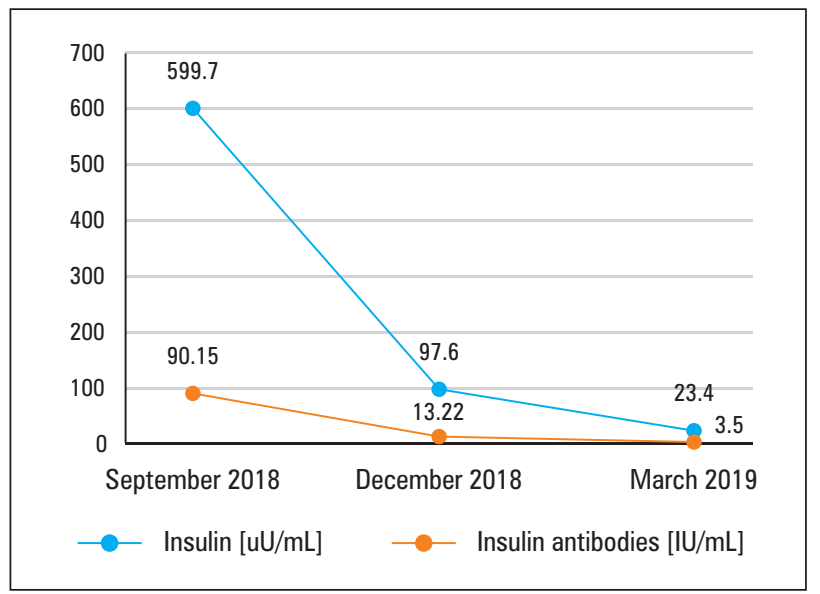

Figure 2. Insulin and insulin autoantibody titres during the follow-up

After discharge she did not experience any hypoglycaemia episodes. The insulin autoantibody titres and Cpeptide levels during the follow-up are shown in Figure 2.

\section{Discussion}

We described ALA-related IAS, a rare autoimmune disorder characterised by spontaneous hypoglycaemia, excessive serum insulin and insulin autoantibody levels, without exposure any exogenous insulin [1].

Insulin autoimmune syndrome is common in Asian countries. It is associated with genetic predisposition, particularly HLA-DR4. In the Japanese population mainly DRB1*0406 and sometimes DRB1*0403 are prone to IAS, whereas HLADR ${ }^{*} 0403$ is more common in Caucasians [3]. The incidence is increasing in non-Asian populations due to accelerating awareness and the prescription of related drugs [3].

In genetically predisposed individuals, the IAS is generally triggered by drugs containing sulphur or sulfhydryl groups such as captopril, methimazole, imipenem, propylthiouracil, and d-penicillamine. Alphalipolic acid (ALA), also containing a sulfhydryl group, was first reported to induce IAS in Japan and was subsequently noticed in many other countries $[4,5]$.

Insulin autoimmune syndrome (IAS) is extremely rare in Caucasians. Gullo et al. analysed six ALA-associated IAS, with HLA-DRB1*04:03 allele in five and HLA-DRB1* 04:06 only in one [5]. In Europe, the low incidence of IAS may be related with the low prevalence of HLA-DRB1*04:06.

The sulfhydryl group-containing drugs may induce insulin autoantibody production [1]. These endogenous antibodies bind to insulin and proinsulin receptors and cause prolonged pancreatic insulin and C-peptide secretion. Insulin and C-peptide secretions continue until the binding capacity of circulating insulin antibodies passes over. Active free insulin increases and lowers blood glucose to hypoglycaemia levels [5].

Certain HLA groups are associated with IAS. Although, HLA-DRB1*0406 is more frequent in Japanese, IAS in Europe shows a correlation with DRB1*04:03 allele that has a high prevalence in Caucasians [5]. In our patient, HLA- DRB1*04:03 and ALA exposure played a role in the development of IAS.

Clinical symptoms of IAS vary from mild cases to severe hypoglycaemia. In contrast to insulinoma that is presented mostly with adrenergic symptoms, IAS usually causes postprandial hypoglycaemia associated with neuroglycopaenic symptoms. Also, insulinoma is accompanied by much lower insulin levels than IAS whereas C-peptide levels are almost equivalent [1].

In most cases, discontinuation of the drug reduces the hypoglycaemia episodes, even though the total remission time may last for three months.

Because ALA consumption is increasing worldwide, clinicians should be aware of the potential interactions the drug can cause. Among patients using sulfhydryl-containing drugs and presenting with hypoglycaemia and extremely high levels of insulin, IAS should be considered rather than insulinoma.

\section{Conflict of interest}

No potential conflict of interest was reported by the authors.

\section{Disclosure}

The authors declared that this study received no financial support.

\section{Patient consent}

Written, informed consent was obtained from the patient.

\section{Author contributions}

N.O. - conceptualisation; C.A. S.S. - data curation; M.S., Y.S — formal analysis: A.O., N.O — methodology: C.A., S.S. — software: M.S., Y.S. — investigation; N.O., A.O. - writing (original draft); N.O., Y.S. — writing (review \& editing).

References

1. Ismail AA. The insulin autoimmune syndrome (IAS) as a cause of hypoglycaemia: an update on the pathophysiology, biochemical investigations and diagnosis. Clin Chem Lab Med. 2016; 54(11): 1715-1724, doi: 10.1515/cclm-2015-1255, indexed in Pubmed: 27071154.

2. Packer L, Cadenas E. Lipoic acid: energy metabolism and redox regulation of transcription and cell signaling. J Clin Biochem Nutr. 2011; 48(1): 26-32, doi: 10.3164/jcbn.11-005FR, indexed in Pubmed: 21297908.

3. Uchigata Y, Hirata Y, Iwamoto Y. Insulin autoimmune syndrome (Hirata disease): epidemiology in Asia, including Japan. Diabetol Int. 2010; 1(1): 21-25, doi: 10.1007/s13340-010-0001-z.

4. Izzo V, Greco C, Corradini D, et al. Insulin autoimmune syndrome in an Argentine woman taking $\alpha$-lipoic acid: A case report and review of the literature. SAGE Open Med Case Rep. 2018; 6: 2050313X18819601, doi: 10.1177/2050313X18819601, indexed in Pubmed: 30627435.

5. Gullo D, Evans JL, Sortino G, et al. Insulin autoimmune syndrome (Hirata Disease) in European Caucasians taking $\alpha$-lipoic acid. Clin Endocrinol (Oxf). 2014; 81(2): 204-209, doi: 10.1111/cen.12334, indexed in Pubmed: 24111525. 